University of Nebraska - Lincoln

DigitalCommons@University of Nebraska - Lincoln

Robert Katz Publications

Research Papers in Physics and Astronomy

June 1980

\title{
Supralinearity of Peak 5 and Peak 6 in TLD-700
}

Michael P. R. Waligórski

Institute of Nuclear Physics, Krakow, Poland, z5waligo@cyf-kr.edu.pl

Robert Katz

University of Nebraska-Lincoln, rkatz2@unl.edu

Follow this and additional works at: https://digitalcommons.unl.edu/physicskatz

Part of the Physics Commons

Waligórski, Michael P. R. and Katz, Robert, "Supralinearity of Peak 5 and Peak 6 in TLD-700" (1980). Robert Katz Publications. 130.

https://digitalcommons.unl.edu/physicskatz/130

This Article is brought to you for free and open access by the Research Papers in Physics and Astronomy at DigitalCommons@University of Nebraska - Lincoln. It has been accepted for inclusion in Robert Katz Publications by an authorized administrator of DigitalCommons@University of Nebraska - Lincoln. 
Published in Nuclear Instruments and Methods 172 (1980), pp. 463-470. Copyright (C) 1980 North-Holland Publishing Company (Elsevier). Used by permission. http://www.sciencedirect.com/science/journal/0168583X

Research for this paper was supported by the United States Department of Energy.

Submitted December 21, 1979.

\title{
Supralinearity of Peak 5 and Peak 6 in TLD-700
}

\author{
Michael P. R. Waligórski \\ University of Nebraska-Lincoln, Lincoln, NE 68588, USA (Permanent address: Institute of Nuclear Physics, Krakow, Poland)
}

Robert Katz

Behlen Laboratory of Physics, University of Nebraska-Lincoln, Lincoln, NE 68588 USA

\begin{abstract}
Track theory has been applied to an earlier suggestion, that the supralinearity of TLDs at high gamma-ray doses is due to pre-existing 1-hit and 2-hit trap structures, to calculate high-LET response. Measured ${ }^{60}$ Co dose responses for peaks 5 and 6 in LiF (TLD700) were decomposed to yield parameters characterizing each peak as a two-component $c$-hit mixture. One value of "trap radius" was assigned to each two-component representation, different for peak 5 and for peak 6 , to calculate their responses for $\mathrm{H}$, $\mathrm{He}, \mathrm{C}, \mathrm{O}$, and Ne bombardments. Calculations reproduce experimental features of the heavy ion response of TLD-700, and provide a means of connecting the gamma- and high-LET responses, in TLDs.
\end{abstract}

\section{Introduction}

Theories attempting to explain supralinearity observed in thermoluminescent dosimeters (TLDs) are typically concerned with trap creation [1], track interaction [2], and pre-existing c-hit trap structures [3]. The present work extends the last concept and explores its implications for heavy-ion response.

We describe the response of a c-hit detector to gamma-rays by two parameters - the hittedness, $c$, and the characteristic dose, $E_{0}$, of gamma-rays at which there is an average of one hit per sensitive element. To calculate the response of such a detector to heavy charged particles we also need to know a third parameter-the radius of the sensitive element, $a_{0}$. We take $a_{0}$ to be related to the size of the sensitive region around a trapping site. The radial distribution of local dose around the path of a heavy ion is then used as a transfer function, relating the low-LET response of a detector to its high-LET response [4].

In earlier work [3] it was suggested that the supralinear response of TL materials for gamma irradiations can be represented by a sum of c-hit components, implying the existence of corresponding (but otherwise undefined) trap structures in this detector. In support of this concept, we note the near-quadratic (2-hit) X-ray response found by Crittenden et al. [5] for peaks 5 and 6 in $\mathrm{BDH} \mathrm{LiF}$ doped with 80 ppm Mg and $\mathrm{Ti}$, measured at a ramp speed of $20^{\circ} \mathrm{C} \mathrm{min}-1$. Peak 6 in the material doped with 3 ppm Ti and measured at $420 \mathrm{~nm}$ wavelength appears to have a near 2-hit response saturating at about $800 \mathrm{krad}$.

Decomposition of experimentally measured responses of TLDs to gamma- or X-rays can yield the values of hittedness, characteristic dose, and relative contribution of each component, but cannot provide us with any information on the size of the sensitive element. The value of $a_{0}$ must be inferred from the response to high-LET radiations.

The response of $\mathrm{LiF}$ to fast charged particles has been measured for protons, alpha-particles, and some heavier ions (C, O, and Ne) for TLD-100 [6, 7] and TLD-700 [8]. The efficiency of LiF, if plotted as a function of the stopping power of the charged particle, appears to be of the order of that for ${ }^{60} \mathrm{Co}$ at lower values of LET and then to decrease with increasing LET [6-10].

Enhancement of the ratio of heights of peak 6 to peak 5 is observed for LiF irradiated with alpha-particles $[9,10]$ and fast neutrons [II], compared with the ratio for gamma-irradiation. A decrease of supralinearity and loss of sensitivity, relative to gamma- irradiation, are seen for LiF (TLD-700) exposed to 3.7 $\mathrm{MeV}$ alpha-particles and $13.3 \mathrm{MeV}$ protons stopping in the material [10]. 
The aim of this work was to investigate the differences in the supralinearity of peak 5 and peak 6 in $\mathrm{LiF}$ (TLD-700) after gamma-ray exposure and to relate them to differences in the high-LET responses of the two peaks reported in the published experimental work. Measurements of ${ }^{60} \mathrm{Co}$ response for peaks 5 and 6 in TLD-700 have already been reported [12], but not for doses at which peak 6 saturates. In the present work, we used a wider dose range and made measurements at two ramp speeds to test the effect of read-out speed on peak separation. In the subsequent calculations, we represent peak 5 and peak 6 by individual two-component $c$-hit mixtures and find their parameters by decomposing the measured ${ }^{60} \mathrm{Co}$ responses of both peaks. The choice of values of $a_{0}$ is made to best fit the available data on relative efficiency and supralinearity for heavy ion irradiations of TLD-700.

It appears that the presented theory is able to reproduce many of the experimental findings for $\mathrm{LiF}$ (TLD-700) and to provide some insight into the relation between gamma- and high-LET responses in this material.

\section{Measurement of ${ }^{60} \mathrm{Co}$ response for peaks 5 and 6 in TLD-700}

Virgin LiF chips (TLD-700 ribbon, 1/4" × 1/4" × 0.035", supplied by HARSHAW) were exposed to ${ }^{60} \mathrm{Co}$ at doses ranging from $3 \mathrm{rad}$ to $10 \mathrm{Mrad}$. A radiotherapy source was used for the $3 \mathrm{rad}-30 \mathrm{krad}$ range with dose rates (20-300) rad $\mathrm{min}^{-1}$, and a $7 \mathrm{KCi}$ source used for the $3 \mathrm{krad}-10 \mathrm{Mrad}$ range, with a dose rate $6 \mathrm{krad}$ $\mathrm{min}^{-1}$. Flat plexiglass containers holding up to 16 chips, of wall thickness $6 \mathrm{~mm}$ on both sides, were exposed in air. All chips were post-irradiation annealed for $10 \mathrm{~min}$ at $90^{\circ} \mathrm{C}$. The HARSHAW $2000 \mathrm{~A}$ and B system (S-13 type photocathode spectral response and a blue filter) was used for read-out. Glow curves were always plotted, at ramp speeds $5^{\circ} \mathrm{C} \mathrm{s}^{-1}$ and $5.5^{\circ} \mathrm{C}$ $\mathrm{min}^{-1}$. For certain high exposure readouts using the faster ramp, the blue filter was replaced by a neutral density filter (Melles Griot 03 FNQ 077) of effective density $D=3.58$, causing no apparent change in the shape of the glow curves. Examples of glow curves plotted using the slower ramp are shown in Figure 1. The heights of peak 5 and 6 were read from glow curves without any background subtraction, normalized to their respective saturation values and plot- ted as a function of dose. The shapes of dose dependences for a given peak appeared not to depend on the ramp speeds used, therefore data could be reduced to two sets describing dose responses of peak 5 and peak 6, shown in Figure 2. Each data point represents $8-6$ readouts and has a relative standard deviation error of $4 \%-10 \%$.

The dose responses of peak 5 and peak 6 are quite different. For peak 5 supralinearity sets in above 300 rad and peak height saturates at about $100 \mathrm{krad}$. In our measurements of peak 6 , supralinearity is observed above $30 \mathrm{rad}$, the lowest dose at which this peak could be distinguished in the glow curve (see Figure 1), and saturation occurs at about $600 \mathrm{krad}, \mathrm{Al}-$ though supralinearity for this peak is clearly higher than that for peak 5, the quadratic dependence found by Crittenden et al. [5] is not observed in our measurements. However, saturation for peak 6 occurs at doses similar to those quoted by Crittenden et al. for BDH LiF doped with 80 ppm Mg and 3 ppm Ti.

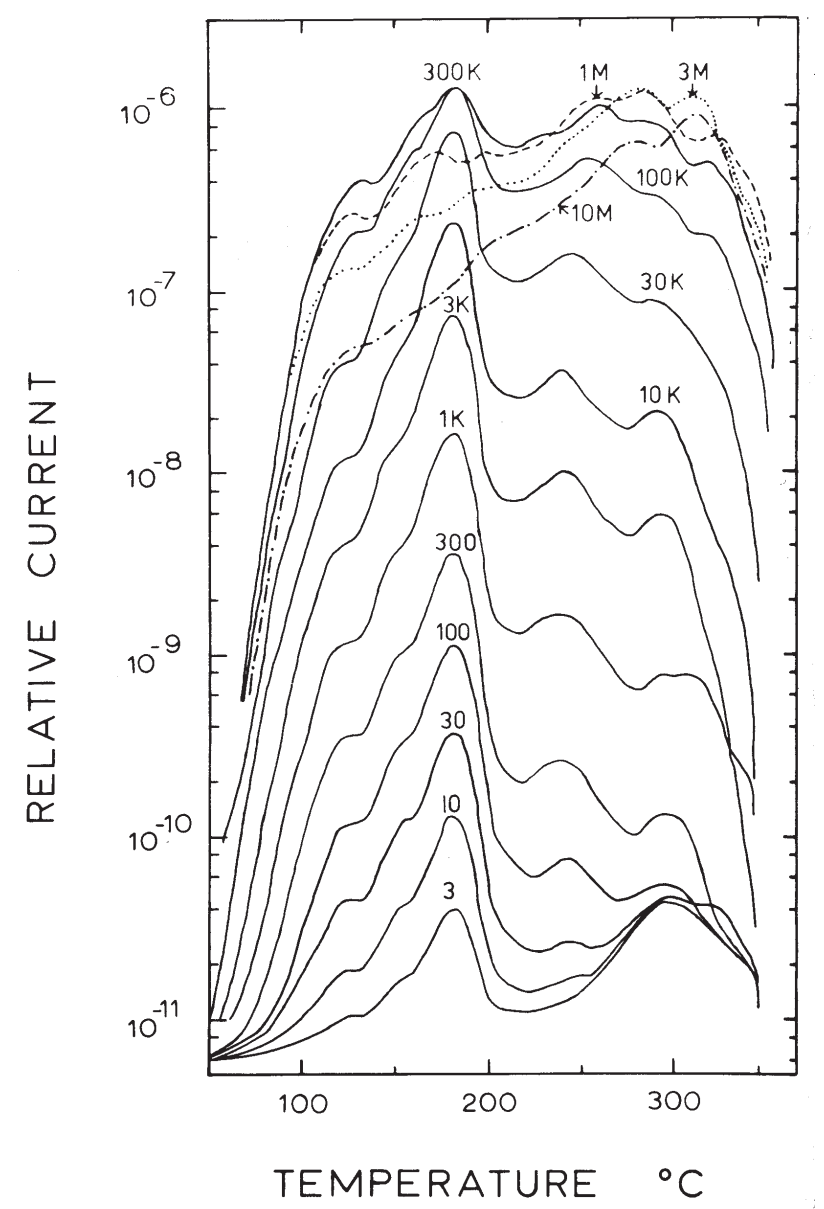

Figure 1. Glow curves plotted at ramp speed $5.5^{\circ} \mathrm{C} \mathrm{min}^{-1}$ for TLD-700 exposed to ${ }^{60} \mathrm{Co}$. Dose range 3 rad to $3 \mathrm{Mrad}$, dose for each curve in rads. 


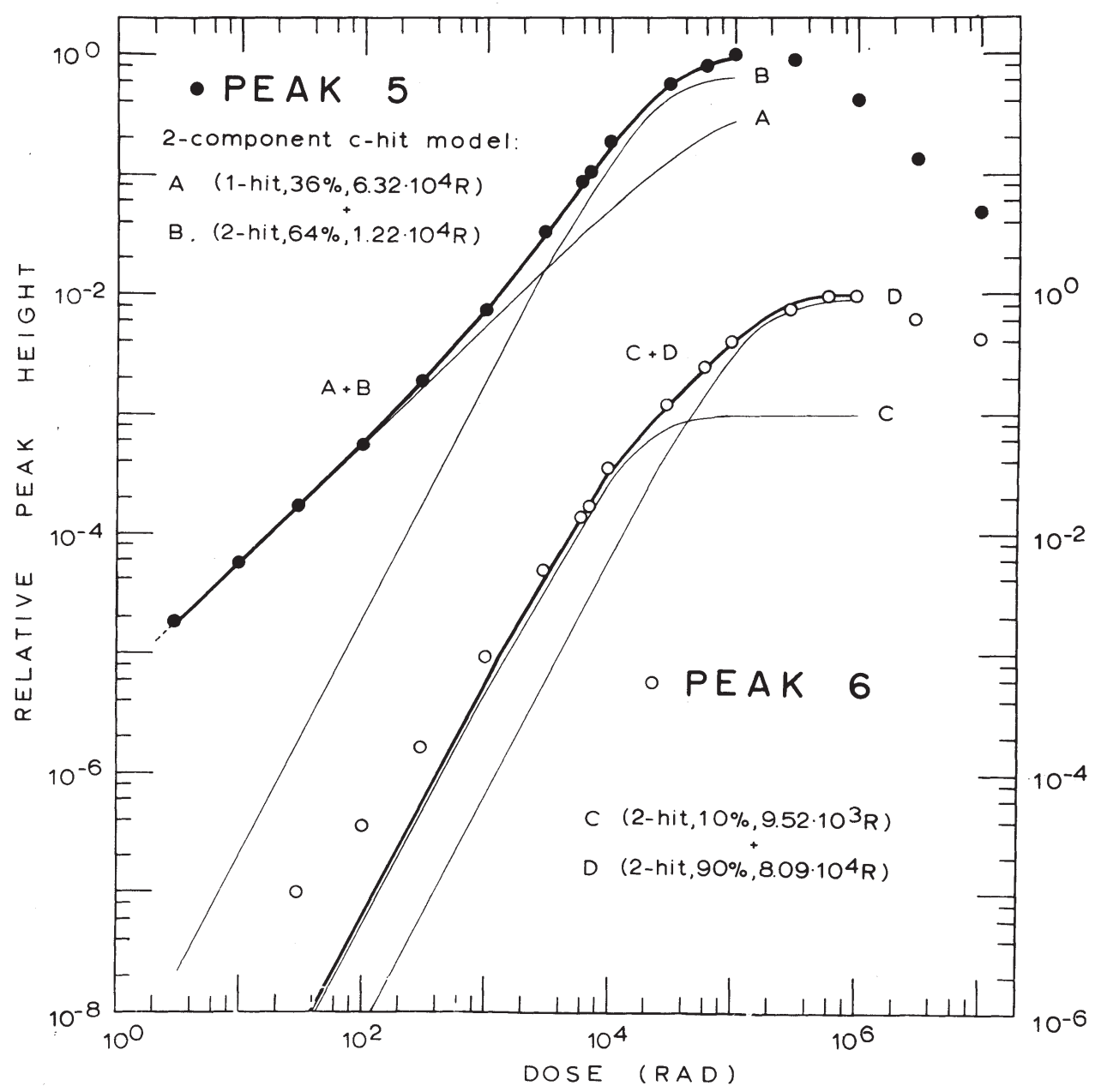

Figure 2. Gamma-ray dose response for peak 5 and peak 6 in TLD-700. Peak heights normalized to saturation values at doses: 100 krad for peak 5, and 1 Mrad for peak 6. Heavy lines through experimental points are respective two-component $c$-hit mixtures representing peak $5(\mathrm{~A}+\mathrm{B})$ and peak $6(\mathrm{C}+\mathrm{D})$, light lines show individual components. Best fitted values of hittedness, percent contribution, and characteristic dose (rads), are indicated for each component.

\section{Theory of the two-component $c$-hit detector}

The principle of calculating the response of a $c$-hit detector to gamma- and heavy-ion irradiations has been extensively discussed in earlier works (see references [3] and [4] and the references therein). The present calculation concerns a two-component $c$-hit detector in which each $c$-hit component is characterized by its own set of parameters - hittedness, characteristic dose, and relative contribution, i.e. $\left(c_{1}, E_{01}\right.$, $R)$ and $\left[c_{2}, E_{02}(1-R)\right]$, respectively. Only 1-hit and 2-hit detectors are considered here, therefore the cumulative Poisson distribution used to describe the response of a detector after a uniform dose $D$ of gamma rays [4], reduces to:

$$
P(c=1, A)=1-\mathrm{e}^{-A} \quad \text { for 1-or-more hits, }
$$

and

$$
P(c=2, A)=1-(1+A) \mathrm{e}^{-\mathrm{A}} \text { for 2-or-more hits, }
$$

where $P(c, A)$ is the probability that $c$ or more hits are observed in a target where $A\left(=D / E_{0}\right)$ is the average number of hits per target.

The TL signal (peak height) after a dose $D$ of uniform gamma-irradiation for a two-component $c$-hit detector, normalized to saturation, is:

$$
\begin{aligned}
\mathrm{TL}(D) & =R P\left(c=c_{1}, D / E_{01}\right) \\
& +(1-R) P\left(c=c_{2}, D / E_{02}\right) .
\end{aligned}
$$

The response of a two-component $c$-hit detector after a fluence $F$ (particles $\mathrm{cm}^{-2}$ ) of heavy particles of charge $Z$, relative velocity $\beta$, and stopping power $L$, is calculated by summing the responses $k_{1}$ and $k_{2}$ of each component, multiplied by their respective contributions, $R$ and $(1-R)$. Calculations are made for 
water, for track segment bombardment [4], i.e. for constant $\beta$ and $L$, which corresponds experimentally to particles passing through a thin detector. In principle, a different value of $a_{0}$ can be associated with each c-hit component, however, to limit the number of fitted parameters, a single value off $a_{0}$ was assumed for both components. The calculation is outlined for a detector consisting of a 1-hit component and a 2hit component with characteristic doses $E_{01}$ and $E_{02}$, respectively.

First, we calculate the response for the 1-hit component, $k_{1}$.

The dose deposited by the ion beam is:

$$
D_{\mathrm{i}}=F L
$$

and the corresponding TL signal (normalized to its value at saturation for gamma-rays) is given by the probability of activating the 1-hit sensitive element:

$$
k_{1}=1-\mathrm{e}^{-\sigma_{1} F}=1-\mathrm{e}^{-\sigma_{1} D_{\mathrm{i}} / L}
$$

where $\sigma_{1}$ is the single-particle activation cross-section, calculated as a function of $Z, \beta, a_{0}$, and $E_{01}$ :

$$
\sigma_{1}=\int_{\tau_{\min }}^{\tau_{\max }+a_{0}} 2 \pi t P\left(c=1, \frac{\bar{E}(t, \beta, Z)}{E_{01}}\right) \mathrm{d} t .
$$

In the above expression, $t$ is the radial distance from the ion's path, $\tau_{\min }=10^{-2} \AA, \tau_{\max }$ is the maximum range of $\delta$-rays in the detector medium, and $\bar{E}(t, \beta, Z)$ is the average dose $\left(\mathrm{erg} \mathrm{cm}^{-3}\right)$ deposited by $\delta$-rays in a sensitive element of radius $a_{0}$, the center of which is at distance $t$. For a given detector medium, the number of $\delta$-rays depends on $z^{2} / \beta^{2}$ of the ion, where $z$ is the effective charge number of the ion of atomic number $Z$ moving at relative speed $\beta$ through the medium. (For further details, see reference [4] and references therein.)

The dose response, $k_{1}\left(D_{\mathrm{i}}\right)$, for a 1-hit detector is linear up to saturation, therefore the 1-hit part of a twocomponent detector cannot introduce supralinearity into the overall dose curve for heavy-ion irradiation at any value of LET.

The calculation for the 2-hit part of a two-component $c$-hit detector is more complicated, as it must include ion-kill and gamma-kill modes of sensitive element inactivation [4].

We first introduce the "saturation cross-section," $\sigma_{0}:$

$$
\sigma_{0}=1.18 \pi a_{0}^{2}
$$

which corresponds to the geometrical cross-section multiplied by a factor accounting for the "brush" of $\delta$-rays surrounding the path of a heavy ion. The procedure for finding the value of this parameter is described elsewhere for $m$-target detectors [4]. Our value is a result of a similar procedure developed for c-hit detectors.

Next, the single-particle inactivation cross-section is calculated for the 2-hit cumulative Poisson distribution:

$$
\sigma_{2}=\int_{\tau_{\min }}^{\tau_{\max }+a_{0}} 2 \pi t P\left(c=2, \frac{\bar{E}(t, \beta, Z)}{E_{02}}\right) \mathrm{d} t,
$$

where symbols retain their meaning from equation (6). The fraction of the dose deposited by the beam which contributes to the ion-kill mode of sensitive element inactivation, $P_{\mathrm{I}}$, is given by the ratio:

$$
P_{\mathrm{I}}=\sigma_{2} / \sigma_{0}
$$

The probability, $\Pi_{i}$, that a sensitive element survives (is not activated) in the ion-kill mode after a fluence $F$ of beam particles, is:

$$
\Pi_{\mathrm{i}}=\mathrm{e}^{-\sigma_{2} F}
$$

The fraction of the beam dose which contributes to sensitive element inactivation through the gammakill mode is $\left(1-P_{\mathrm{I}}\right) F L$ and the probability, $\Pi_{\gamma}$, that a sensitive element survives in that mode is given from the 2-hit cumulative Poisson distribution as:

$$
\Pi_{\gamma}=1-P\left(c=2, \frac{\left(1-P_{\mathrm{I}}\right) F L}{E_{02}}\right) .
$$

The surviving fraction, $N / N_{0}$, of an initial population of $N_{0}$ sensitive elements after a fluence $F$ of beam particles is the product of survival probabilities after ionkill and gamma-kill modes of inactivation:

$$
N / N_{0}=\Pi_{i} \Pi_{r}
$$

The probability that a sensitive element is activated is then $\left(1-N / N_{0}\right)=\left(1-\Pi_{\mathrm{i}} \Pi_{\gamma}\right)$, and the corresponding response (normalized to the gamma-ray saturation value) contributed by the second component of the detector is:

$$
k_{2}=\left(1-\Pi_{i} \Pi_{\gamma}\right) .
$$

The signal due to the ion-kill mode increases linearly with dose, and that due to gamma-kill increases quadratically. Thus, the shape of the dose response, $k_{2}(D i)$, depends on the value of $P_{1}$, i.e. on the value of $\sigma_{2}$ with respect to $\sigma_{0}$. If $\sigma_{2}$ is close to (or exceeds) the saturation value ("track-width regime"), the ion-kill 
mode dominates, and supralinearity disappears. If $\sigma_{2}$ is small compared with $\sigma_{0}$ ("grain- count regime"), we expect to see some residual supralinearity in the dose-response curve.

Finally, the TL signal of a two-component $(1+2)$ hit detector after a fluence $F$ of beam particles is obtained by adding the responses of each component, multiplied by their respective contributions:

$$
\mathrm{TL}\left(D_{\mathrm{i}}\right)=R k_{1}+(1-R) k_{2}
$$

The TL signal after a fluence $F$ of beam particles for a $(2+2)$-hit detector is calculated in the same manner, by repeating equations (7-13) for both components and substituting $E_{02}$ with $E_{01}$ where appropriate $\left(P_{I^{\prime}}\right.$ $\Pi_{i}$, and $\Pi \gamma$ have to be calculated separately for each component).

The overall dose response, $\operatorname{TL}\left(D_{\mathrm{i}}\right)$, for a two-component $c$-hit detector depends in a complicated way on detector and beam parameters. It is worth noting that it is only from the ion-kill mode contribution in the 2-hit component that the overall efficiency with respect to gamma-irradiation can exceed 1 . Whether or not this actually occurs depends on the current parameter values. In general, we expect the efficiency of the 2-hit component to reach maximum at $z^{2} / \beta^{2}=$ $2 \kappa$, where $\kappa=E_{0} a_{0}^{2}\left(2 \times 10^{-7} \mathrm{erg} \mathrm{cm}^{-1}\right)$, for water, and $E_{0}$ and $a_{0}$ correspond to respective values for the 2hit component [4]. We shall see later that this property could be exploited to estimate experimentally the value of $a_{0}$ in the two-component representation of peak 5 in TLD-700 from measurements of efficiency as a function of $z^{2} / \beta^{2}$ (or LET) for proton or alphaparticle bombardments.

\section{Decomposition of measured gamma-ray responses}

Equation (3) was used to determine the values of $c_{1}, c_{2}, E_{01}, E_{02}$ and $R$, by assuming the values of $c_{1}$ and $c_{2}$ [i.e. defining the functional relation, according

Table 1. Best fitted values of characteristic dose, $E_{0}$, and relative contribution, $R$, for 1-hit and 2-hit components representing peak 5 and peak 6 in TLD-700.

\begin{tabular}{llllll}
\hline Peak & 1-hit & & & 2-hit & \\
\cline { 2 - 3 } \cline { 5 - 6 } & $R$ & $E_{0}(\mathrm{rad})$ & & $R$ & $E_{0}(\mathrm{rad})$ \\
\hline peak 5 & 0.360 & $6.323 \times 10^{4}$ & & 0.640 & $1.215 \times 10^{4}$ \\
peak 6 & & & 0.102 & $9.522 \times 10^{3}$ \\
& & & 0.898 & $8.094 \times 10^{4}$ \\
\hline
\end{tabular}

to equations (1) and (2)] and treating the remaining three variables as free parameters in computer fits to experimental data.

A $(1+2)$-hit mixture was assumed for peak 5 , and the following expression was fitted to the experimental points:

$$
\begin{aligned}
P_{5}(D) & =R\left(1-\mathrm{e}^{-D / E_{01}}\right) \\
& +(1-R)\left[1-\left(1+D / E_{02}\right) \mathrm{e}^{-D / E_{02}}\right] .
\end{aligned}
$$

Best fitting values of $E_{01}, E_{02}$, and $R$ are listed in Table 1. The decomposition into 1-hit and 2-hit parts is shown in Figure 2.

Because of its weakness with respect to peak 5, data for peak 6 were more difficult to analyze. Judging from the shape of the glow curves presented in Figure 1, significant background contributions are visible at lower doses, below 1 krad. A $(2+2)$-hit mixture was assumed for peak 6 and the following expression fitted to data points in the range $3 \mathrm{krad}-1 \mathrm{Mrad}$ :

$$
\begin{aligned}
P_{6}(D)= & R\left[1-\left(1+D / E_{01}\right) \mathrm{e}^{-D / E_{01}}\right] \\
& +(1-R)\left[1-\left(1+D / E_{02}\right) \mathrm{e}^{-D / E_{02}}\right] .
\end{aligned}
$$

Value of $E_{01}, E_{02}$, and $R$ best fitting the data in the limited dose range are listed in Table 1 and the decomposition is shown in Figure 2.

Our analysis suggests the existence of four (or possibly three) trap structures which contribute to the measured dose dependence for peaks 5 and 6 in TLD-700. It is not improbable that the dominating 2hit component in peak 5 and the small contribution $(10 \%)$ to peak 6 are both due to the same trap structure, as indicated by the relatively close values of $E_{0}$ for these components.

\section{Calculation of heavy ion response}

Parameters of the two-component representations of peak 5 and peak 6 in TLD-700, listed in Table 1 were used in all subsequent calculations of the heavy ion response in TLD-700. For a given bombardment (specified by $Z, \beta$, and LET), a complete dose response over a range of fluences was calculated for each peak, using several values of $a_{0}$. The calculated curves are presented as efficiencies, relative to ${ }^{60} \mathrm{Co}$, at a specified dose, $D_{0}$, and as plots of supralinearity versus dose. The relative supralinearity index, $S_{\text {rel, }}$ is defined as follows:

$$
S_{\text {rel }}(D)=S(D) / S\left(D_{0}\right)
$$


Table 2. Experimental [8] and calculated values of efficiency, relative to ${ }^{60} \mathrm{Co}$, for peak 5 for TLD-700 irradiated with heavy ions

\begin{tabular}{llllcc}
\hline $\begin{array}{l}\text { Ion } \\
\text { species }\end{array}$ & $\begin{array}{l}\text { Energy } \\
\left(\mathrm{MeV} \mathrm{amu}^{-1}\right)\end{array}$ & $\begin{array}{l}\text { Water LET } \\
\left(\mathrm{MeV} \mathrm{cm}^{-1}\right)\end{array}$ & $\begin{array}{l}\text { Efficiency relative to } \\
\text { experiment } \\
\text { ref. [8] }\end{array}$ & $a_{0}=50 \AA$ & $a_{0}=100 \AA$ \\
\hline $\mathrm{C}$ & 252 & $1.41 \times 10^{2}$ & $0.89 \pm 0.02$ & 0.78 & 0.91 \\
$\mathrm{O}$ & 300 & $2.25 \times 10^{2}$ & $0.82 \pm 0.05$ & 0.76 & 0.83 \\
$\mathrm{O}$ & 1050 & $1.42 \times 10^{2}$ & $0.90 \pm 0.05$ & 0.78 & 0.90 \\
$\mathrm{Ne}$ & 372 & $3.18 \times 10^{2}$ & $0.73 \pm 0.03$ & 0.74 & 0.79 \\
\hline
\end{tabular}

where

$$
S(D)=\mathrm{TL}(D) / D, \quad S\left(D_{0}\right)=\mathrm{TL}\left(D_{0}\right) / D_{0^{\prime}}
$$

and $\operatorname{TL}(D)$ and $\operatorname{TL}\left(D_{0}\right)$ are the signals (peak height) at doses $D$ and $D_{0}$, respectively.

\subsection{Peak 5}

The value of $a_{0}$ for the two-component representation of peak 5 was estimated from a best fit to the experimental results of Patrick et al. [8], who measured relative efficiencies of TLD-700 ribbon exposed with $\mathrm{C}, \mathrm{O}$, and $\mathrm{Ne}$ ions of energies above $250 \mathrm{MeV}^{\mathrm{amu}}{ }^{-1}$, i.e. at conditions satisfying the theoretical calculation. Efficiencies at $D_{0}=10 \mathrm{rad}$ were calculated for peak 5 using values of $a_{0}=50,100$, and $150 \AA$, and compared with experimental values. We assume that in the overall signal measured experimentally, the small contribution from peak 6 may be neglected. Experiment and theory are compared in Table 2 . On the basis of this comparison, relative efficiencies of peak 5 were calculated for the three values of $a_{0}$, for $\mathrm{H}, \mathrm{He}$, $\mathrm{C}, \mathrm{O}$, and $\mathrm{Ne}$ ion bombardments over a range of LET values (from $\beta=0.98$ until the Bragg peak) for each ion. Results of the calculation are presented in Figure 3, together with experimental data points from the work of Patrick et al. [8] and of Wingate et al. [6]. Experimental points for stopping alpha-particles are plotted at their initial value of LET. It is interesting to note that the calculation predicts efficiencies exceeding 1 for proton and alpha-particle irradiations over ranges of LET dependent on the value of $a_{0}$. Possibly, this could provide means of estimating $a_{0}$ experimentally. Tochilin et al. [7] report efficiencies exceeding 1 , for TLD-100. As LET increases, for slow particles in the vicinity of the Bragg peak, in all cases efficiency falls radically.

The relative supralinearity index, calculated for ${ }^{60} \mathrm{Co}$, a relativistic proton, and a range of alpha-par- ticle bombardments, is plotted as a function of dose in Figure 4A. In qualitative agreement with experimental findings of Tochilin et al. [7] and of Wingate et al. [6], the supralinearity for the relativistic proton is identical with that of ${ }^{60} \mathrm{Co}$, and then gradually decreases with increasing LET of the bombarding particle. A dramatic change from supralinearity to sublinearity at high doses is seen in the calculations for alpha-particle energies between 1000 and $100 \mathrm{MeV}$. As the particle's energy further decreases, the curves become linear for increasingly higher doses.

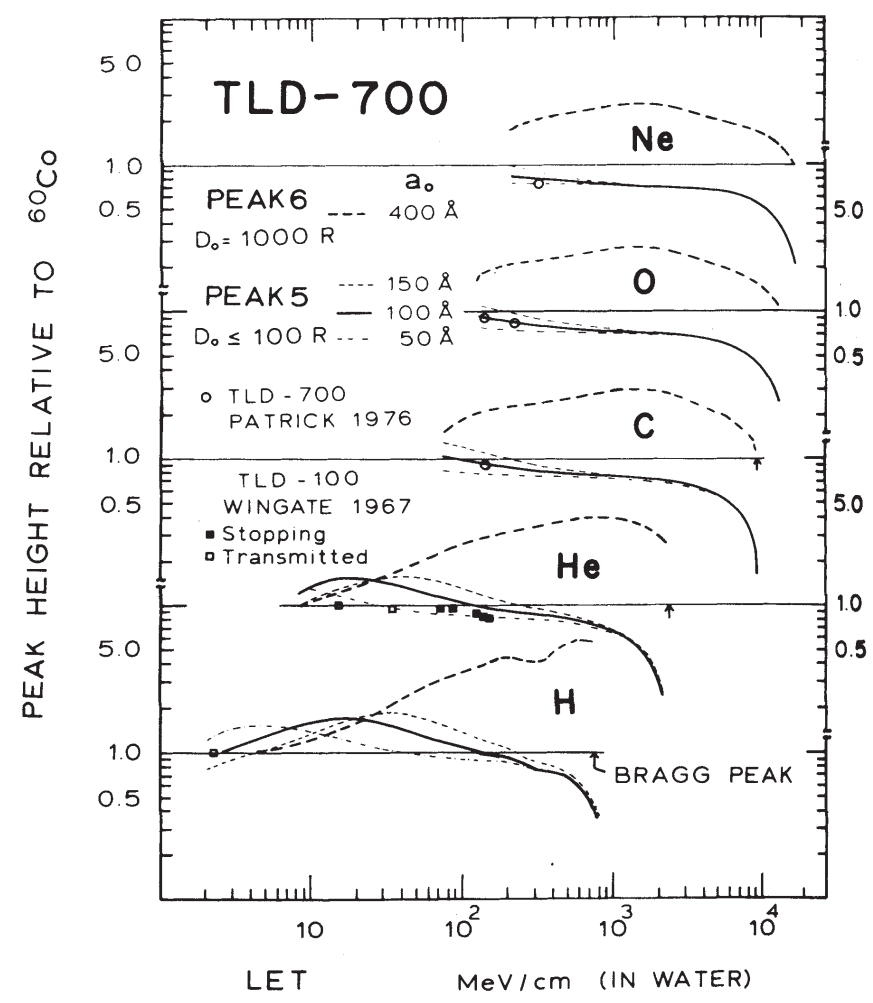

Figure 3. Calculated efficiencies, relative to ${ }^{60} \mathrm{Co}$, versus LET (in water) for $\mathrm{H}, \mathrm{He}, \mathrm{C}, \mathrm{O}$, and $\mathrm{Ne}$ ion bombardments. For peak 5 , results shown for $a_{0}=50,100$, and $150 \AA$, and efficiencies calculated at $D_{0}=10 \mathrm{rad}$. For peak $6, a_{0}=400 \AA$, efficiencies calculated at $D_{0}=1 \mathrm{krad}$. 


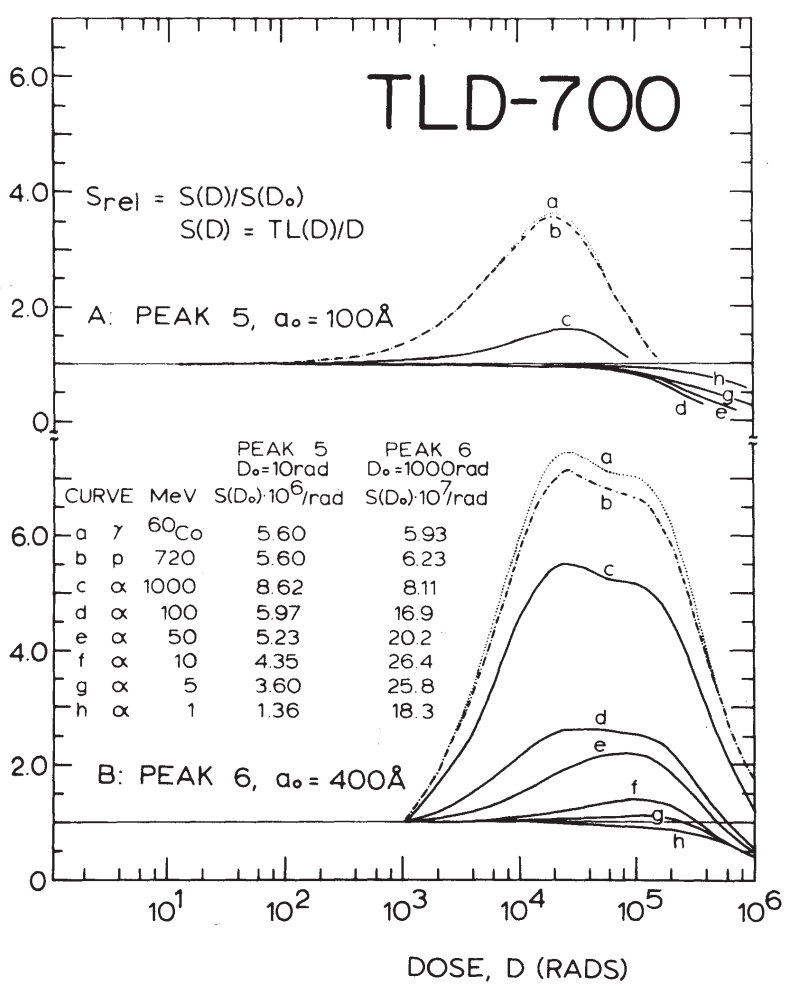

Figure 4. Relative supralinearity index versus dose, calculated for ${ }^{60} \mathrm{Co}$, relativistic proton $(720 \mathrm{MeV})$, and alpha-particle bombardments (1000-1 MeV). A: Peak 5, $a_{0}=100 \AA$, plots normalized to 1 at $D_{0}=10 \mathrm{rad}$. B: Peak $6, a_{0}=400 \AA$, plots normalized to 1 at $D_{0}=1 \mathrm{krad}$. Normalizing factors listed. For a given bombardment, the peak height at dose $D_{0}, \mathrm{TL}\left(D_{0}\right)$, is equal to $D_{0} S\left(D_{0}\right)$.

\subsection{Peak 6}

The experimental observation by Jahnert [10] of a residual supralinearity in TLD-700 ribbon irradiated with alpha-particles of energy $3.7 \mathrm{MeV}$ and lower, stopping in the material, was used to estimate the value of $a_{0}$ for peak 6 . At the same time, Jahnert observes an increase in the ratio of heights of peak 6 to peak 5. We presume that if the TL signal is measured by integrating the glow curves over temperatures covering both peaks, then the supralinearity observed is due to the supralinearity of peak 6 . The choice of $a_{0}$ equal to $400 \AA$ for the two-component representation of that peak was made to give a calculated "threshold" of supralinearity for $5 \mathrm{MeV}$ alphaparticle bombardment.

Due to the problem of background in the two-component representation of ${ }^{60} \mathrm{Co}$ response for peak 6, relative efficiencies and supralinearity were calculated at $D_{0}=1000 \mathrm{rad}$, for the same heavy-ion bombardments used to calculate the response for peak 5 .
Results of these calculations are presented in Figure 3 and Figure 4B.

Immediately apparent from Figure 3 is the much higher relative efficiency for peak 6 as compared with that of peak 5, especially at high values of LET (low energies) for proton and alpha-particle bombardments. As expected, supralinearity for peak 6 is much higher than that for peak 5, as seen in Figure 4. These two results of the theoretical calculation suggest an explanation for the enhancement in the peak height ration and increase in sensitivity, observed experimentally in LiF irradiated with alpha-particles and fast neutrons [9-11].

\section{Discussion}

The presented calculation illustrates the manner in which track theory relates the gamma-ray and heavyion responses in TLD-700. We are able to reproduce, at least qualitatively, most of the experimental observations concerning this detector. For some of the experimental data, quantitative agreement is achieved. However, several difficulties remain.

Use of the slow ramp speed for glow curve measurements, although improving the overall resolution somewhat, did not lead to a better separation between peak 5 and peak 6 , nor did it change the shapes of their ${ }^{60} \mathrm{Co}$ responses. Possibly, different doping could result in a better separation, enabling us to assign trap parameters with greater confidence.

Peaks 5 and 6 in TLD-700 are represented in the calculation by two-component $c$-hit structures, implying that two types of traps contribute to each peak. It appears that at least three different traps would have to be present in our LiF sample: (1) a 1-hit trap with $E_{0} \simeq 60 \mathrm{krad}$, (2) a 2-hit trap with $E_{0} \simeq 10 \mathrm{krad}$, and (3) a 2-hit trap with $E_{0} \simeq 80 \mathrm{krad}$. It is not unlikely that the X-ray response observed by Crittenden et al. [5] for, peak 6 in $\mathrm{BDH} \mathrm{LiF}$ doped with $80 \mathrm{ppm} \mathrm{Mg}$ and 3 ppm Ti which saturates at doses similar to ours, is due to the dominating 2-hit trap in our decomposition of the same peak.

It is not evident that both traps contributing to peak 5 should have the same value of $a_{0}$. This was assumed, strictly for reasons of simplicity, in the calculations of heavy-ion response for that peak. Similar assumption made in the calculations for peak 6, bears less influence on the results, as one trap clearly dominates in our decomposition for that peak (see Table 1). 
It is interesting to speculate on the physical meaning off $a_{0}$. If we take the composition of our sample to be that of the material quoted by Crittenden et al., then $\mathrm{Mg}$ and $\mathrm{Ti}$ impurities (80 ppm and 3 ppm, respectively) would occur roughly every 25 and 70 lattice spacings, i.e. every 100 and $300 \AA$ (the cubic lattice constant in $\mathrm{LiF}$ is $\simeq 4 \AA$ ). It would be tempting to conclude that the near agreement with our estimates of $a_{0}$ for peaks 5 and 6 is not entirely fortuitous, especially as our values are estimated in a "water TLD." The uncertainty with which we determine our values of $a_{0}$ for peak 5 and peak 6 does not warrant the use of a "scaling factor" due to the density of LiF (as the density of $\mathrm{LiF}$ is $2.64 \mathrm{~g} \mathrm{~cm}^{-3}$, this "factor" is $2.64^{1 / 3} \simeq$ 1.4 , by which our values of $a_{0}-100 \AA$ and $400 \AA$, respectively - might be divided).

If any significance is to be attached to the results of heavy-ion response calculations, the prediction that the relative efficiency for peak 5 in TLD-700 exceeds 1 , should be relatively easy to verify experimentally. A systematic measurement of efficiency in the range of LET values $10-100 \mathrm{MeV} \mathrm{cm}{ }^{-1}$ for proton or alphaparticle bombardments could test our calculations and yield a better indication of the value of $a_{0}$ (based on the value of $z^{2} / \beta^{2}$ at which the efficiency reaches a maximum) for the sensitive element dominating in peak 5 .

We stress that our predictions of the response of peak 5 and peak 6 to heavy-ion bombardments are somewhat speculative and that their validity can be tested only after experimental measurements are performed for a variety of ion bombardments over a wide range of energies.

\section{Conclusions}

We have proposed a theoretical calculation relating the low-LET and high-LET response of TLD-700, based on the assumption that 1-hit and 2-hit traps (presumably related to $\mathrm{Mg}$ and $\mathrm{Ti}$ impurities) preexist in this material. We do not know the dopant concentration in our sample, nor are we able to assign values of trap parameters with confidence. A systematic measurement of the response of TLD-700 to proton and alpha-particle bombardment could provide us with a further clarification of the two-component $c$-hit representation of peaks 5 and 6 .
It would be however more advantageous if TL materials showing a "clean" quadratic X- or gammaray response were investigated and developed, perhaps by suitable variation of the known and controlled dopant composition. The presented theoretical model of the variation of TLD response with LET could then be tested. Such a material would also offer us the best opportunity to achieve discrimination between gamma-rays and neutrons or other high-LET radiations.

The original c-hit detector computer codes were developed by R. A. Krauter. The LMCHOL optimization code developed in Argonne National Laboratory by K. Hilstrom was used for parameter fitting. We thank A. S-F. Li and Y-L. Chang for adapting these programs to perform our calculations. We also thank P. Dettman, M.D., and Dr. B. Maxcy for permission to use their ${ }^{60} \mathrm{Co}$ irradiation facilities.

\section{References}

[1] J. R. Cameron, N. Suntharalingam, and G. N. Kenney, Thermoluminescent Dosimetry (University of Wisconsin Press, Madison, 1968).

[2] F. H. Attix, in: Proc. 4th Int. Conf. on Luminescence Dosimetry, Krakow, Poland 1974, p. 31.

[3] L. Larsson and R. Katz, Nucl. Instr. and Meth. 138 (1976) 631.

[4] R. Katz, Nucl. Track Detect. 2 (1978) 1.

[5]G. C. Crittenden, P. D. Townsend, J. Gilkes, and M. C. Wintersgill, J. Phys. D: Appl. Phys. 7 (1974) 2410.

[6] C. L. Wingate, E. Tochilin, and N. Goldstein, in: Proc. Int. Conf. on Luminescence Dosimetry, Stanford 1965, p. 421.

[7] E. Tochilin, N. Goldstein, and J. T. Lyman, in: Proc. 2nd Int. Conf. on Luminescence Dosimetry, Gatlinburg, Tennessee 1968, p. 424.

[8] J. W. Patrick, L. D. Stephens, R. H. Thomas, and L. S. Kelly, Health Phys. 30 (1976) 295.

[9] A. C. Lucas and C. Rainbolt, in: Proc. 2nd Int. Conf. on Luminescence Dosimetry, Gatlinburg, Tennessee 1968, p. 456

[10] B. Jahnert, Health Phys. 23 (1972) 112.

[11] K. Ayyangar, A. R. Reddy, and G. L. Brownell, in: Proc. 2nd Int. Conf. on Luminescence Dosimetry, Gatlinburg, Tennessee 1968, p. 525.

[12] E. W. Mason and G. S. Linsley, in: Proc. 3rd Int. Conf. on Luminescence Dosimetry, Risø, Denmark 1971, p. 164. 